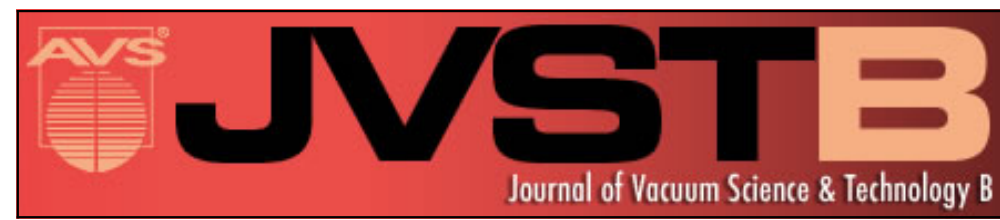

\title{
Study of substrate-directed ordering of long double-stranded DNA molecules on bare highly oriented pyrolytic graphite surface based on atomic force microscopy relocation imaging
}

Huabin Wang, Hongjie An, Feng Zhang, Zhixiang Zhang, Ming Ye, Peng Xiu, Yi Zhang, and Jun Hu

Citation: Journal of Vacuum Science \& Technology B 26, L41 (2008); doi: 10.1116/1.2968698

View online: http://dx.doi.org/10.1116/1.2968698

View Table of Contents: http://scitation.aip.org/content/avs/journal/jvstb/26/5?ver=pdfcov

Published by the AVS: Science \& Technology of Materials, Interfaces, and Processing

\section{Articles you may be interested in}

Self-assembly of $50 \mathrm{bp}$ poly $(\mathrm{dA}) \cdot \mathrm{poly}(\mathrm{dT}) \mathrm{DNA}$ on highly oriented pyrolytic graphite via atomic force microscopy observation and molecular dynamics simulation

J. Chem. Phys. 139, 085102 (2013); 10.1063/1.4818595

Stability, resolution, and ultra-low wear amplitude modulation atomic force microscopy of DNA: Small amplitude small set-point imaging

Appl. Phys. Lett. 103, 063702 (2013); 10.1063/1.4817906

Improved Kelvin probe force microscopy for imaging individual DNA molecules on insulating surfaces Appl. Phys. Lett. 97, 203703 (2010); 10.1063/1.3512867

Ultrafast molecule sorting and delivery by atomic force microscopy

Appl. Phys. Lett. 88, 183105 (2006); 10.1063/1.2195777

High-resolution imaging of single-stranded DNA on mica surface under ultrahigh vacuum conditions by noncontact atomic force microscopy

J. Vac. Sci. Technol. B 17, 1941 (1999); 10.1116/1.590853

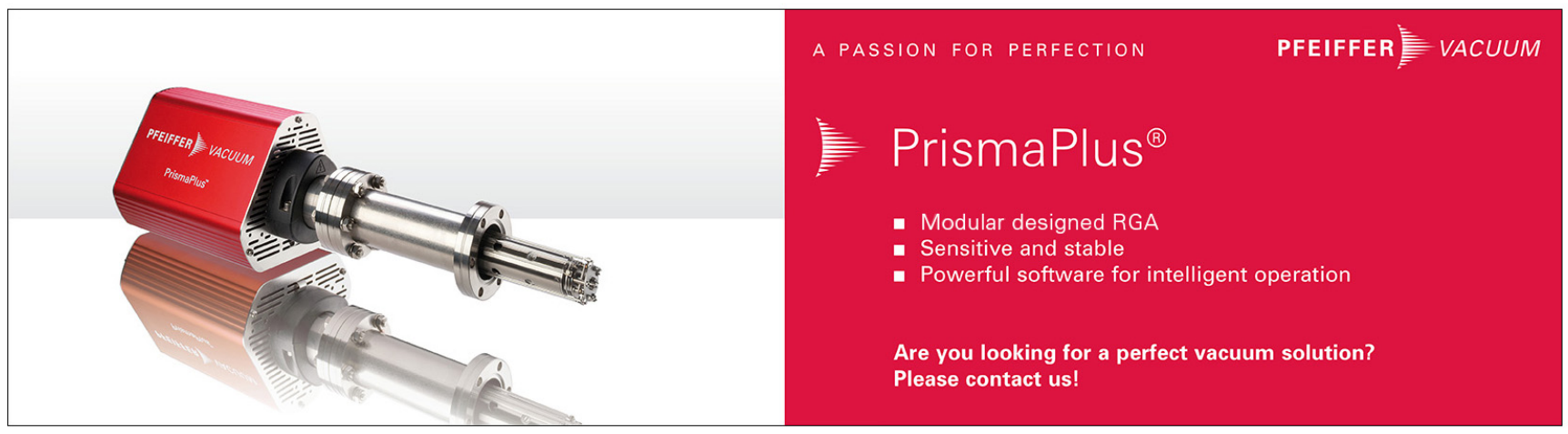




\title{
Study of substrate-directed ordering of long double-stranded DNA molecules on bare highly oriented pyrolytic graphite surface based on atomic force microscopy relocation imaging
}

\author{
Huabin Wang \\ Shanghai Institute of Applied Physics, Chinese Academy of Sciences, Shanghai 201800, People's Republic \\ of China and Graduate School of the Chinese Academy of Sciences, Beijing 100039, People's \\ Republic of China \\ Hongjie An \\ Bio-X Life Science Research Center, Shanghai Jiao Tong University, Shanghai 200030, People's Republic \\ of China
}

Feng Zhang, Zhixiang Zhang, Ming Ye, and Peng Xiu

Shanghai Institute of Applied Physics, Chinese Academy of Sciences, Shanghai 201800, People's Republic of China and Graduate School of the Chinese Academy of Sciences, Beijing 100039, People's

Republic of China

Yi Zhanga)

Shanghai Institute of Applied Physics, Chinese Academy of Sciences, Shanghai 201800, People's Republic of China

Jun $\mathrm{Hu}^{\text {b) }}$

Shanghai Institute of Applied Physics, Chinese Academy of Sciences, Shanghai 201800, People's Republic of China and Bio-X Life Science Research Center, Shanghai Jiao Tong University, Shanghai 200030, People's Republic of China

(Received 26 March 2008; accepted 14 July 2008; published 8 September 2008)

\begin{abstract}
Usually, long double-stranded DNA molecules exhibit an aggregated or a random spreading behavior when deposited on a highly ordered pyrolytic graphite (HOPG) substrate. In this article, the authors report a novel phenomenon where randomly oriented DNA strands can gradually be rearranged into two-dimensional ordered nanostructures under the operation of repeatedly rotating a water droplet on the DNA sample. The process of DNA rearrangement was traced by using atomic force microscopy relocation imaging. The orientation of the ordered DNA strands shows a threefold symmetry consistent with the underlying atomic lattice of the HOPG substrate, signifying a substrate-directed ordering process. The relevant mechanism is discussed. () 2008 American Vacuum Society. [DOI: 10.1116/1.2968698]
\end{abstract}

\section{INTRODUCTION}

In recent years, exploiting inorganic solid surfaces to direct the construction of ordered and functional molecular complexes has attracted much attention. This is because the resultant structures can be fine controlled at the molecular level and delicately aligned with respect to the atomic lattices of the underlying substrates, which provides new solutions for "bottom-up" fabrication. ${ }^{1-3}$ Much progress has been made on the substrate-directed arrangement of various materials such as peptides, ${ }^{1,4-6}$ copolymers, ${ }^{7}$ oligonucleotides, ${ }^{8}$ and ionic liquid ${ }^{9}$ to form functional thin films, microcrystals, or ordered nanostructures.

Double-stranded DNA (ds-DNA), a uniform onedimensional molecule with a diameter of $\sim 2 \mathrm{~nm}$, is widely accepted as a smart material for nanotechnology. Previously, long ds-DNA was successfully used as a template to fabricate nanowires ${ }^{10,11}$ field-effect transistors ${ }^{12}$ and quantum interference device. ${ }^{13}$ Thus, substrate-directed organization of

a)Electronic mail: yzhang@sinap.ac.cn

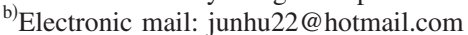

long ds-DNA on a solid substrate is envisioned as promising applications for large-scale bottom-up construction. On the other hand, ordering of long DNA molecules on a solid substrate [e.g., on a bare highly ordered pyrolytic graphite (HOPG) surface] may open up new opportunities for the direct sequencing or mapping of DNA molecules. ${ }^{14,15}$

Long ds-DNA can also serve as an excellent model system to investigate the mechanism of substrate-directed ordering of long molecules. Unlike small molecules, substratedirected ordering of larger molecules on a surface is significantly hindered by the molecules' severe steric randomness and stronger interaction with the substrate. ${ }^{16}$ In principle, the registry between larger molecules and a surface is much more unfavorable entropically. Only if an external energy is provided to the system can a high degree of registry occur. ${ }^{17}$ For example, many experiments have shown that even on well-defined solid surfaces, long DNA molecules usually tend to form random features during sample preparation. ${ }^{18-22}$ By using the "molecular combing" method or its modifications, ${ }^{23-25}$ long DNA molecules can be stretched almost along one direction, but the substrate's lattice orientation cannot be achieved. Recently, poly(sodium- 


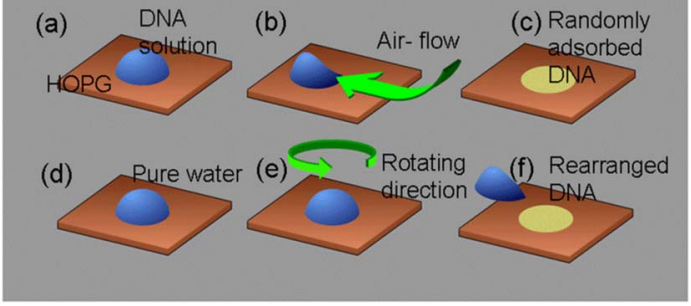

FIG. 1. Schematic representation of the protocol for constructing oriented nanostructures of long ds-DNA molecules on a HOPG surface.

4-styrenesulfonate) macromolecules have been observed aligned along the lamella of self-assembled alkylamine on the HOPG surface. ${ }^{26}$ So far, it is still uncertain whether a bare solid substrate can be used to direct the ordering of long DNA molecules.

In this article, based on atomic force microscopy (AFM) relocation imaging, we report that, by rotating a water droplet on the DNA sample, we are able to transform the randomly deposited long ds-DNA molecules into oriented twodimensional (2D) patterns on the bare HOPG surface.

\section{EXPERIMENT}

The original Lambda DNA solution (Sino-American Biotechnology Company) was diluted to $10 \mathrm{ng} / \mu$ l with Milli-Q water (18.2 M $\Omega$ ) prior to use. The HOPG (grade ZYH, MikroMash Co.) was freshly cleaved with adhesive tape before use. All images were collected with tapping-mode AFM (Nanoscope IIIa Multimode, Veeco Instruments Inc.) equipped with a $\mathrm{J}$ or $\mathrm{E}$ scanner in air under relative humidity of $30 \%-40 \%$ at room temperature. Silicon cantilevers (NSC35/Al BS or NSC35/Pt-Ti, MikroMash Co.) had a typical force constant of $4.5 \mathrm{~N} / \mathrm{m}$ and resonant frequency of $150 \mathrm{kHz}$.

The protocol for ordering DNA nanostructures consists of three steps (Fig. 1). (i) A drop of $10 \mu \mathrm{l}$ DNA solution (10 ng/ $\mu \mathrm{l}$ ) was deposited onto a freshly cleaved HOPG substrate $\left(1 \times 1 \mathrm{~cm}^{2}\right)$ attached to the axis of a rotary device. The droplet was allowed to incubate for $3 \mathrm{~min}$ (a) and then gently blown off with airflow [(b) and (c)]. (ii) A drop of $10 \mu \mathrm{l}$ Milli-Q water was deposited onto the HOPG surface at the exact location where the DNA droplet was previously deposited (d). After rotating the device at a certain velocity (e) for 3-5 min, the droplet was thrown off quickly from the substrate by abruptly speeding the rotation to a higher velocity (f). Ordered DNA patterns could be formed on the HOPG surface during this process. (iii) The second step could be repeated several times to refine the DNA pattern.

The AFM relocation imaging was achieved using the following procedures. (i) After the initial image was collected, the tip was retracted for one step (several microns). (ii) A transparent clean film was covered and fixed on the screen of the charge-coupled device combined with the AFM system, and the characteristics of the sample surface and probe cantilever were delineated on the film by using a watercolor pen. (iii) Before imaging the treated sample, the remounted

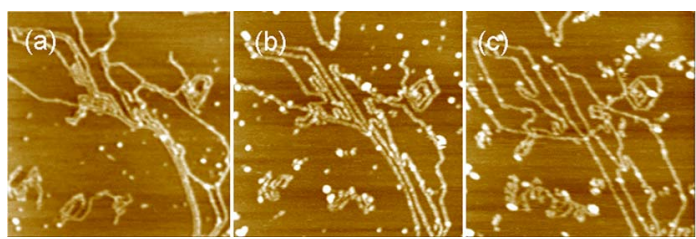

FIG. 2. AFM images of randomly deposited DNA molecules (a) and reordered DNA molecules [(b) and (c)]. The image size is $460 \times 460 \mathrm{~nm}^{2}$.

sample and probe cantilever were regulated to superpose the corresponding features recorded on the transparent film. (iv) A relatively large scan area (usually several microns) was first imaged, and the previous target location could be zoomed into later. In this way, we could easily and precisely repeatedly relocate the same small area.

\section{RESULTS AND DISCUSSION}

After step (i) of our protocol for ordering DNA, DNA molecules were randomly deposited on the HOPG surface [Fig. 2(a)]. This result is consistent with those reported in previous literature. ${ }^{18,19}$ However, subsequent operations [step (ii)] resulted in the rearrangement of DNA molecules. As shown in Fig. 2(b), the DNA strands were straightened and reshaped into oriented patterns on the surface, in which most of the DNA strands are either parallel to or have an angle of about $60^{\circ}$ or $120^{\circ}$ with each other. More importantly, the DNA features could be further refined into more-ordered patterns step by step if we repeated the operation [step (iii)], as shown in Fig. 2(c).

Interestingly, we often observed exquisite hexagonal patterns consisting of individual DNA fragments/molecules. For example, as shown in Fig. 3, the orientations of the DNA strands clearly show a threefold symmetry, which is confirmed by the Fourier transform of the image (inset in Fig. 3). Short sections where the ds-DNA molecules melted into two

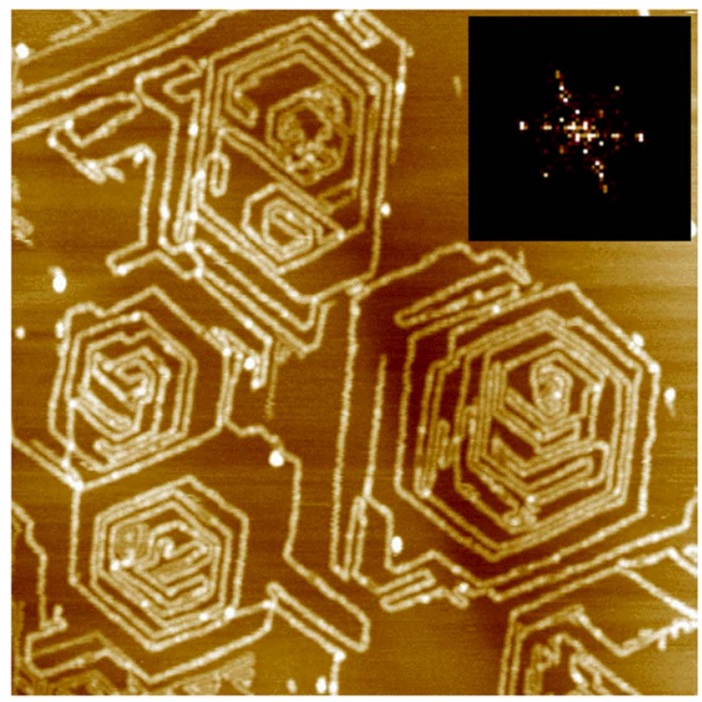

FIG. 3. AFM image of ordered DNA patterns formed on the HOPG surface. The scan size is $800 \times 800 \mathrm{~nm}^{2}$. The inset is the $2 \mathrm{D}$ Fourier transform of the image. 


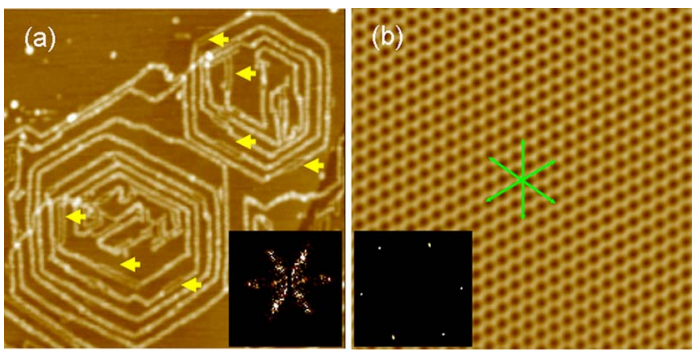

FIG. 4. Images reveal the correlation between oriented DNA strands and the atomic lattice of HOPG. (a) AFM image of ordered DNA patterns. The inset is the $2 \mathrm{D}$ Fourier transform of the image. The yellow arrows indicate that some parts in ds-DNA were separated into two single DNA strands. (b) STM image of the atomic lattice of the underlying HOPG. The inset is the 2D Fourier transform of the image. The green arrows indicate the orientation of HOPG lattice. The two images of $2 \mathrm{D}$ Fourier transform clearly show a similar threefold symmetry. The STM image was obtained by using the Nanoscope IIIa instrument in a constant-current mode with an E scanner and a $\mathrm{Pt} / \mathrm{Ir}\left(10 \%\right.$ Ir) tip. The scan sizes are $490 \times 490 \mathrm{~nm}^{2}$ in (a) and 8 $\times 8 \mathrm{~nm}^{2}$ in (b)

single strands could sometimes be observed in the DNA patterns [Fig. 4(a)]. The presence of the single-stranded DNA fragments is not a result of diluting original DNA solution by Milli-Q water before use since similar phenomena were not found on other substrates such as a mica surface. The orientations of the DNA strands in the patterns correlate well with the atomic lattice of the HOPG substrate underneath [Fig. 4(b)], indicating a substrate-directed ordering process that leads to an energetically preferred alignment of the DNA strands on the HOPG lattices. This is similar to the assembly of alkyl chains on the HOPG surface. ${ }^{27}$ It is worth noting that the ordered DNA molecules adsorbed on the HOPG in our experiments were stable under continued AFM scanning. No obvious locational dependence was observed for the formation of the patterns at present. We found that short DNA strands, such as 886 bp DNA strands $(\sim 300 \mathrm{~nm})$, could easily be washed off by water during the manipulation. However, the DNA fragments with a length of about $2 \mu \mathrm{m}$ could still be ordered with this technique (data not shown).

We found that the rotation velocity could strongly affect the pattern formation. The appropriate velocity was 600-1200 rpm in our experiments, which would result in ordering of roughly $30 \%$ DNA strands after one operation. Operation at a velocity below $300 \mathrm{rpm}$ resulted in little change of the DNA features, even with a long period of time (e.g., $20 \mathrm{~min}$ ). Nevertheless, beyond $1800 \mathrm{rpm}$, the droplet flew off the substrate quickly because of the centrifugal force, and the 2D DNA patterns were barely formed. Obviously, during the rotating process, a viscous force was generated by water near the substrate surface, ${ }^{28}$ which was exerted on the adsorbed DNA and mediated them to move around slightly on the surface until the DNA formed a pattern with the minimum-energy state, as suggested for other molecules registered with the substrates.2, ${ }^{29}$ Previously, Yokota et $a l .{ }^{28}$ and Kim et al. ${ }^{30}$ investigated the interactions between fluid flow and DNA molecules in a droplet deposited onto a substrate during the spinning process. From theoretical modeling, they found that the obtained forces were not consistent with the results from the experiments. In this study, the complex movements of DNA molecules at the solid-liquid interface make an in-depth interpretation of the results very difficult at present. On the other hand, the "specific" interactions between DNA molecules and the HOPG substrate should also be emphasized, which we believe is the intrinsic factor that determined the "template-directed" arrangement of DNA molecules on the surface. Previous studies have shown that the breathing fluctuations of dsDNA could expose some bases, ${ }^{31,32}$ and the exposed hydrophobic core would interact with the underlying hydrophobic substrates such as HOPG (Refs. 18 and 19) and poly(dimethylsiloxane $)^{24,30}$ through hydrophobic interactions. Indeed, we did find that some ds-DNA fragments were separated into two single strands on the substrate. From an intuitive point of view, only when the DNA molecules covered the rows of the most densely packed carbon atoms could the maximum contact between the exposed bases and carbon atoms form. Such an interaction may order DNA with an orientation that matches the threefold crystallographic symmetry of the substrate, similar to the mechanism proposed previously for the arrangement of peptide chains with hydrophobic residues on graphite. ${ }^{6}$ We anticipate that computer simulation methods would be helpful to gain a deeper understanding of the ordering mechanism of DNA molecules on HOPG in the future.

It is necessary to point out that the stable arrangement of ordered DNA strands on the bare HOPG surface is important for those who want to image DNA with scanning tunneling microscopy $^{33}$ (STM) for higher spatial resolution or electrical property measurement. Almost two decades ago, oriented barlike features on freshly cleaved HOPG surface were found and have been thought to mimic DNA and other biomolecules in the STM studies. ${ }^{34}$ In our case, however, the oriented structures are definitely DNA molecules, based both on our relocating studies and the widely accepted characteristic features of DNA molecules in AFM (i.e., a uniform polymer with an apparent height around $0.7 \mathrm{~nm}$ in air by tapping-mode AFM). ${ }^{35}$ Because the intimate contact between the DNA molecules and graphene structures are relatively stable and may render interesting electronic properties, ${ }^{14}$ we believe that such ordered DNA structures would facilitate direct sequencing or mapping DNA molecules with highresolution methods such as STM (Ref. 36) and fluorescent microscopy. ${ }^{15}$

\section{CONCLUSIONS}

We presented a novel method capable of ordering long ds-DNA molecules into oriented patterns on a bare HOPG surface. This technique is distinct from previous approaches $^{24-26,37-39}$ for fabricating DNA patterns and may be used as prominent templates for assembling nanomaterials. Moreover, with this technique, artifact-free, perfectly dispersed, and well-straightened DNA samples can be prepared on the bare graphite surface with a higher stability, which will provide a new opportunity for the direct sequencing or mapping of DNA molecules. ${ }^{14,15}$ 


\section{ACKNOWLEDGMENTS}

This work was supported by the National Natural Science Foundation of China (No. 10335070), the Science and Technology Ministry of China (Nos. 2006AA04Z309 and 2007CB936003), the Chinese Academy of Sciences (Nos. KJCX2.YW.M03 and KJCX2.Y.W.H06).

${ }^{1}$ C. L. Brown, I. A. Aksay, D. A. Saville, and M. H. Hecht, J. Am. Chem. Soc. 124, 6846 (2002).

${ }^{2}$ D. E. Hooks, T. Fritz, and M. D. Ward, Adv. Mater. (Weinheim, Ger.) 13, 227 (2001).

${ }^{3}$ J. Y. Cheng, A. M. Mayes, and C. A. Ross, Nat. Mater. 3, 823 (2004).

${ }^{4}$ X. G. Liu, Y. Zhang, D. K. Goswami, J. S. Okasinski, K. Salaita, P. Sun, M. J. Bedzyk, and C. A. Mirkin, Science 307, 1763 (2005).

${ }^{5}$ F. Zhang et al., Angew. Chem., Int. Ed. 45, 3611 (2006).

${ }^{6}$ T. Kowalewski and D. M. Holtzman, Proc. Natl. Acad. Sci. U.S.A. 96, 3688 (1999)

${ }^{7}$ S. A. Prokhorova et al., Langmuir 16, 6862 (2000).

${ }^{8}$ S. I. Tanaka, Y. Maeda, L. T. Cai, H. Tabata, and T. Kawai, Jpn. J. Appl. Phys., Part 1 40, 4217 (2001).

${ }^{9}$ Y. D. Liu, Y. Zhang, G. Z. Wu, and J. Hu, J. Am. Chem. Soc. 128, 7456 (2006).

${ }^{10}$ E. Braun, Y. Eichen, U. Sivan, and G. Ben-Yoseph, Nature (London) 391, 775 (1998).

${ }^{11}$ J. Lund, J. C. Dong, Z. X. Deng, C. D. Mao, and B. A. Parviz, Nanotechnology 17, 2752 (2006).

${ }^{12}$ K. Keren, R. S. Berman, E. Buchstab, U. Sivan, and E. Braun, Science 302, 1380 (2003).

${ }^{13}$ D. S. Hopkins, D. Pekker, P. M. Goldbart, and A. Bezryadin, Science 308, 1762 (2005)

${ }^{14}$ G. Lu, P. Maragakis, and E. Kaxiras, Nano Lett. 5, 897 (2005).

${ }^{15}$ R. Lebofsky and A. B. Bensimon, Funct. Genomic. Proteomic. 1, 385 (2003)

${ }^{16}$ H. Xu, S. S. Sheiko, D. Shirvanyants, M. Rubinstein, K. L. Beers, and K.
Matyjaszewski, Langmuir 22, 1254 (2006).

${ }^{17}$ A. K. Chakraborty and A. J. Golumbfskie, Annu. Rev. Phys. Chem. 52, 537 (2001).

${ }^{18}$ A. M. O. Brett and A. M. Chiorcea, Langmuir 19, 3830 (2003).

${ }^{19}$ X. H. Jiang and X. Q. Lin, Electrochem. Commun. 6, 873 (2004).

${ }^{20}$ Z. F. Shao, News Physiol. Sci. 14, 142 (1999).

${ }^{21}$ X. F. Zhou, Y. Zhang, H. B. Wang, D. X. Niu, P. Q. Wang, and J. Hu, Chin. Phys. Lett. 24, 2692 (2007).

${ }^{22}$ H. B. Wang, X. F. Zhou, H. J. An, Y. C. Guo, J. L. Sun, Y. Zhang, and J. Hu, Chin. Phys. Lett. 24, 644 (2007).

${ }^{23}$ D. Bensimon, A. J. Simon, V. Croquette, and A. Bensimon, Phys. Rev. Lett. 74, 4754 (1995).

${ }^{24}$ C. A. P. Petit and J. D. Carbeck, Nano Lett. 3, 1141 (2003).

${ }^{25}$ J. J. Guan and L. J. Lee, Proc. Natl. Acad. Sci. U.S.A. 102, 18321 (2005).

${ }^{26}$ M. Severin, J. Barner, A. A. Kalachev, and J. P. Rabe, Nano Lett. 4, 577 (2004).

${ }^{27}$ X. Shao, X. C. Luo, X. Q. Hu, and K. Wu, J. Phys. Chem. B 110, 1288 (2006).

${ }^{28}$ H. Yokota, J. Sunwoo, M. Sarikaya, G. V. D. Engh, and R. Aebersold, Anal. Chem. 71, 4418 (1999).

${ }^{29}$ N. J. Reeves and J. S. Evans, J. Phys. Chem. 100, 17297 (1996).

${ }^{30}$ J. H. Kim, W. X. Shi, and R. G. Larson, Langmuir 23, 755 (2007).

${ }^{31}$ A. Hanke and R. Metzler, J. Phys. A 36, L473 (2003).

${ }^{32}$ A. Krueger, E. Protozanova, and M. D. Frank-Kamenetskii, Biophys. J. 90, 3091 (2006).

${ }^{33}$ T. P. Beebe, Jr., T. E. Wilson, D. F. Ogletree, J. E. Katz, R. Balhorn, M. B. Salmeron, and W. J. Siekhaus, Science 243, 370 (1989).

${ }^{34}$ C. R. Clemmer and T. P. Beebe, Jr., Science 251, 640 (1991).

${ }^{35}$ X. J. Li, J. L. Sun, X. F. Zhou, G. Li, P. G. He, Y. Z. Fang, M. Q. Li, and J. Hu, J. Vac. Sci. Technol. B 21, 1070 (2003).

${ }^{36}$ S. M. Lindsay and B. Barris, J. Vac. Sci. Technol. A 6, 543 (1988).

${ }^{37}$ J. Hu, Y. Zhang, H. B. Gao, M. Q. Li, and U. Hartmann, Nano Lett. 2, 55 (2002).

${ }^{38}$ Z. Q. Ouyang, J. Hu, S. F. Chen, J. L. Sun, and M. Q. Li, J. Vac. Sci. Technol. B 15, 1385 (1997).

${ }^{39}$ N. C. Seeman, Nano Lett. 1, 22 (2001) 